\title{
Comparison of Total Body Irradiation (TBI) Conditioning with Non-TBI for Autologous Stem Cell Transplantation in Newly Diagnosed or Relapsed Mature T- and NK-Cell Non-Hodgkin Lymphoma
}

\author{
Chi Hoon Maeng, MD, $\mathrm{PhD}{ }^{1}$ \\ Young Hyeh Ko, MD, PhD² \\ Do Hoon Lim, MD, PhD ${ }^{3}$ \\ Eun Suk Kang, MD, $\mathrm{PhD}^{4}$ \\ Joon Young Choi, MD, PhD ${ }^{5}$ \\ Won Seog Kim, MD, PhD 6 \\ Seok Jin Kim, MD, PhD ${ }^{6,7}$
}

\begin{abstract}
${ }^{1}$ Division of Hemato-Oncology, Department of Internal Medicine, Kyung Hee University Hospital, Kyung Hee University College of Medicine, Seoul, Departments of ${ }^{2}$ Pathology, ${ }^{3}$ Radiation Oncology,

${ }^{4}$ Laboratory Medicine, and ${ }^{5}$ Nuclear Medicine, ${ }^{6}$ Division of Hematology-Oncology, Department of Medicine, Samsung Medical Center, Sungkyunkwan University School of Medicine, Seoul, ${ }^{7}$ Department of Health Sciences and Technology, SAIHST, Sungkyunkwan University, Seoul, Korea
\end{abstract}

\begin{abstract}
Purpose
This retrospective study was conducted for comparison of survival outcomes and toxicities of autologous stem cell transplantation (ASCT) based on the use of total body irradiation (TBI) as a part of the conditioning regimen in patients with mature T-and natural killer (NK)cell lymphomas.
\end{abstract}

\section{Materials and Methods}

Patients who underwent ASCT in the upfront or salvage setting between January 2000 and December 2013 were analyzed. Patients were dichotomized according to the TBI group $(n=38)$ and non-TBI group $(n=60)$ based on the type of conditioning regimen for ASCT.

\section{Results}

Patients with responsive disease underwent upfront ASCT (TBI, n=16; non-TBI, n=29) whereas patients with refractory disease $(T B I, n=9$; non- $T B I, n=12)$ or relapsed disease (TBI, n=13; non-TBI, n=19) underwent ASCT after salvage treatment. Hematologic and non-hematologic toxicities were manageable, and the median cumulative toxicity score according to Seattle criteria was estimated as 2 (range, 0 to 7 ) in both groups. No significant difference in 100-day mortality was observed between the TBI (13\%, 5/38) and non-TBI (12\%, 12/60) groups, and most deaths were related to disease progression. There was no difference in overall and progression-free survival; however, the TBI group showed a trend of better survival in upfront and salvage ASCT than the non-TBI group. However, patients with refractory disease showed the worst outcome regardless of the use of TBI. Patients who showed complete response before ASCT showed better progression-free survival than those who showed partial response.

\section{Conclusion}

TBI could be used as an effective part of conditioning for ASCT in patients with mature T- and NK-cell lymphomas.

\section{Key words}

T-lymphocytes, Nutural killer cells, Lymphoma,

Stem cell transplantation, Whole-body irradiation

\section{Introduction}

High-dose chemotherapy might eradicate residual tumor cells that survive conventional chemotherapy; therefore, high-dose chemotherapy followed by autologous stem cell transplantation (ASCT) has been used as a consolidation treatment for patients with lymphoma. This benefit of ASCT has been proven in a relapse setting; better outcomes were observed for patients with relapsed lymphoma after receiving ASCT than with salvage treatment alone [1]. Based on this demonstrated efficacy of ASCT, upfront ASCT has been recommended for patients with mature T-cell lymphomas due to the high probability of relapse after induction treat- 
ment $[2,3]$. A recent prospective study conducted by the Nordic Lymphoma Group reported encouraging outcomes with upfront ASCT in newly diagnosed T-cell lymphomas [4]. Given that the outcome of ASCT mainly depends on the efficacy of the high-dose chemotherapy used as the conditioning regimen, development of an effective conditioning regimen seems to be important for improving the treatment outcome of ASCT. However, there is no consensus regarding the best conditioning regimen for ASCT in patients with T-cell lymphoma, and previous studies using various conditioning regimens for ASCT reported a 5-year survival outcome of approximately 50\% [5-8]. Total body irradiation (TBI) has been used as a component of the conditioning regimen for ASCT because of its antitumor effect. However, the use of TBI has some limitations due to lack of availability in all transplantation centers and its use might increase toxicity. As a result, TBI was replaced with radiomimetic agents such as busulfan in many conditioning regimens. However, given the radiosensitivity of T-cell lymphoma cells, TBI could still be a useful treatment modality. A previous prospective study reported 3-year overall survival (OS) of $48 \%$ after TBI-containing high-dose chemotherapy followed by upfront ASCT in 83 patients with T-cell lymphoma [9]. A recent retrospective study analyzing the outcome of extranodal natural killer (NK)/T-cell lymphoma patients undergoing upfront ASCT with various conditioning regimens including TBI also reported 3-year OS of 60\% [10]. Despite many studies reporting survival outcomes of ASCT in newly diagnosed as well as relapsed or refractory T-cell lymphoma patients, there were few data regarding the comparison of survival outcomes according to type of conditioning regimens in patients with mature T- and NK-cell lymphomas [11-13]. Therefore, we analyzed consecutive patients who underwent ASCT in the upfront and salvage setting for mature T- and NK-cell lymphomas, and compared survival outcomes based on the use of TBI as a part of the conditioning regimen.

\section{Materials and Methods}

\section{Study design and patients}

This is a retrospective study analyzing survival outcomes of patients with T- and NK-cell lymphoma who underwent ASCT in the upfront or salvage setting. All patients received various high-dose chemotherapy regimens with or without TBI as a conditioning treatment for ASCT. The primary objective was to compare progression-free survival (PFS) after ASCT based on the use of TBI as a part of the conditioning regimen for ASCT. Thus, patients undergoing ASCT after
TBI-containing conditioning regimen were designated as the "TBI group" whereas patients undergoing ASCT without TBI as the "non-TBI group." The secondary objective was to compare the transplantation-related morbidity and mortality between the TBI and non-TBI groups. The other secondary objectives included the comparison of survival outcomes according to the clinical situation at the time of ASCT (upfront ASCT after diagnosis vs. salvage ASCT after relapse), the response to the initial first-line treatment (responsive vs. refractory vs. relapsed disease), the subtype of mature T- and NK-cell lymphomas (T-cell lymphoma vs. extranodal NK/T-cell lymphoma [ENKTL]), and the pretransplantation disease status (complete response [CR] vs. partial response [PR]). The medical records of consecutive patients diagnosed with mature T- and NK-cell lymphoma who underwent ASCT between January 2000 and December 2013 at Samsung Medical Center were reviewed. The inclusion criteria were as follows. First, patients should be pathologically diagnosed with mature T- and NK-cell lymphoma according to the pathology criteria of the World Health Organization [14]. Second, patients received TBI or non-TBIbased high-dose chemotherapy as a conditioning regimen for ASCT. Third, patients received induction chemotherapy with curative intent, with assessment of the response to the induction chemotherapy by conventional computed tomography (CT) and / or positron emission tomography (PET)-CT [15]. This study was approved by the Institutional Review Board of Samsung Medical Center and the requirement for signed informed consent was waived because of its retrospective nature (IRB No. 2015-12-113).

\section{Survival outcomes}

The outcome variables were OS and PFS. OS was defined as the time from the date of ASCT to the date of death from any cause or the date of the last follow-up, and PFS was defined as the time from the date of ASCT to the date of progression or relapse after ASCT or any kind of death including transplantation-related mortality. Living patients were censored on the date of the last follow-up visit, and the final updates of patient survival and disease status were completed at the time of analysis (7 Nov 2015). The pre- and postASCT disease status was assessed using CT and/ or PET-CT. The PET-CT protocol and positive criteria on initial PET-CT scan were previously described in detail [16], and PET-CT was performed using one of two dedicated PET-CT scanners (Discovery LS or Discovery STE, GE Healthcare, Milwaukee, WI) without intravenous or oral contrast material.

\section{ASCT procedure}

Peripheral blood stem cell collection was achieved by 
chemotherapy and granulocyte-colony stimulating factor mobilization. The TBI-based conditioning regimen consisted of etoposide, cyclophosphamide, TBI: etoposide 1,800 $\mathrm{mg} / \mathrm{m}^{2}$ intravenous infusion over 26 hours on day 7 , cyclophosphamide $60 \mathrm{mg} / \mathrm{kg}$ intravenously over 2 hours per day on day 6 to day 4 with infusion of mesna at the same dosage, and 900 cGy of TBI (300 cGy per day from day 3 to day 1). The non-TBI-based conditioning regimens were BEAM (BCNU $300 \mathrm{mg} / \mathrm{m}^{2}$ iv on day 6 , etoposide $200 \mathrm{mg} / \mathrm{m}^{2}$ intravenously over 1 hour per day on day 5 to day 2; total $800 \mathrm{mg} / \mathrm{m}^{2}$, cytarabine $200 \mathrm{mg} / \mathrm{m}^{2}$ intravenously twice daily on day 5 to day 2; total $1,600 \mathrm{mg} / \mathrm{m}^{2}$, melphalan $140 \mathrm{mg} / \mathrm{m}^{2}$ intravenously per day on day 1 ), Bu-Cy-etoposide (busulfan $3.2 \mathrm{mg} / \mathrm{kg}$ intravenously over 3 hours per day on day 7 to day 5 , etoposide $400 \mathrm{mg} / \mathrm{m}^{2}$ intravenously over 3 hours per day on day 5 to day 4 , and cyclophosphamide $50 \mathrm{mg} / \mathrm{kg}$ intravenously over 2 hours per day on day 3 to day 2), BuMel-etoposide (busulfan $3.2 \mathrm{mg} / \mathrm{kg}$ intravenously over 3 hours per day on day 8 to day 6 , etoposide $400 \mathrm{mg} / \mathrm{m}^{2}$ intravenously over 3 hours per day on day 5 to day 4 , and melphalan $50 \mathrm{mg} / \mathrm{m}^{2}$ over 1 hour per day on day 3 to day 2$)$, and Bu-Mel-Thiotepa (busulfan $3.2 \mathrm{mg} / \mathrm{kg}$ intravenously over 3 hours per day on day 8 to day 6 , melphalan $50 \mathrm{mg} / \mathrm{m}^{2}$ intravenously over 1 hour per day on day 5 to day 4 , and thiotepa $250 \mathrm{mg} / \mathrm{m}^{2}$ over 2 hours per day on day 3 to day 2). Physicians decided on the conditioning regimens for each patient after considering the availability of drugs and TBI, and clinical situation of each patient.

\section{Assessment of transplantation-related outcomes}

Neutrophil engraftment was defined as the first 3 continuous days with recovery of absolute neutrophil count (ANC) $>500 / \mathrm{mm}^{3}$ without another decrease. Platelet engraftment was defined as the first 3 continuous days with recovery of platelet count $>20,000 / \mathrm{mm}^{3}$ without a decrease or need for repeat transfusion. Neutrophil engraftment days and platelet engraftment days were defined as the time from the day of transplantation to the first day of neutrophil/ platelet engraftment. Toxicities, including febrile neutropenia and documented bacteremia, were graded according to Common Toxic Criteria of Adverse Events (CTCAE) ver. 4.0 after review of medical records. Regimen-related organ toxicities were assessed in the first 100 days from transplantation and graded according to the Seattle criteria [17]. The cumulative toxicity score was the sum of each score measured in a specific organ during the recovery time after transplantation. The length of hospitalization and 100-day mortality were also compared between the TBI and non-TBI groups. The length of hospitalization was defined as the time between the date of stem cell infusion (day 0 ) and discharge from the hospital and 100-day mortality was defined as any kind of death within 100 days after ASCT.

\section{Statistical analysis}

Fisher exact test was applied to determine associations between categorical variables. The Kaplan-Meier method was used in univariate analysis of survival outcomes and the results were compared using the log-rank test. The toxicity profile was compared using Fisher exact test, and in the case of numeric variables including cumulative toxicity score, the Wilcoxon rank-sum test was performed to examine statistical differences in the sum of the scores between groups (PASW ver. 18, SPSS Inc., Chicago, IL).

\section{Results}

\section{Patients' characteristics}

A total of 38 patients received TBI for ASCT (TBI group) whereas 60 patients only received high-dose chemotherapy (non-TBI group). Their characteristics at diagnosis are summarized in Table 1 . The median age at diagnosis was 43 years (range, 18 to 65 years) for the TBI group and 44 years (range, 16 to 64 years) for the non-TBI group. The median time from diagnosis to ASCT was 0.6 years (range, 0.1 to 6.7 years) and 0.5 years (range, 0.2 to 9.3 years), respectively, for the TBI and non-TBI groups. The comparison of baseline characteristics showed no significant difference between TBI and non-TBI groups except the International Prognostic Index (IPI). Thus, patients with high-intermediate/high risk of IPI accounted for a significantly higher proportion in the TBI group compared to the non-TBI group, although there were more missing data in the TBI group (Table 1). The most common histologic type was ENKTL $(n=47)$ and the remaining types included peripheral T-cell lymphoma, not otherwise specified (PTCL-NOS, $n=23$ ), angioimmunoblastic T-cell lymphoma (AITL, $\mathrm{n}=11$ ), anaplastic large cell lymphoma (ALCL, $\mathrm{n}=11)$, enteropathy-associated T-cell lymphoma $(\mathrm{n}=3)$, subcutaneous panniculitis-like T-cell lymphoma $(\mathrm{n}=2)$, and hepatosplenic $\mathrm{T}$-cell lymphoma $(\mathrm{n}=1)$. Induction chemotherapy was decided on by the physician based on the treatment policy and subtypes of lymphomas. Thus, anthracyclinebased treatments such as CHOP (cyclophosphamide, doxorubicin, vincristine, and prednisone) or CHOP-like regimens were mainly used for patients with PTCL-NOS, AITL, and ALCL whereas non-anthracycline-based regimens such as SMILE (steroid, methotrexate, ifosfamide, L-asparaginase, and etoposide) and VIDL (etoposide, ifosfamide, dexamethasone, and L-asparaginase) were mainly used for 
Table 1. Patient characteristics

\begin{tabular}{|c|c|c|c|}
\hline Characteristic & TBI $(n=38)$ & Non-TBI $(n=60)$ & p-value \\
\hline \multicolumn{4}{|l|}{ Age (yr) } \\
\hline$\leq 60$ & $34(90)$ & $57(95)$ & 0.425 \\
\hline$>60$ & $4(10)$ & $3(5)$ & \\
\hline \multicolumn{4}{|l|}{ Sex } \\
\hline Male & $24(63)$ & $37(62)$ & $>0.99$ \\
\hline Female & $14(37)$ & $23(38)$ & \\
\hline \multicolumn{4}{|l|}{ ECOG grade } \\
\hline $0 / 1$ & $30(79)$ & $53(88)$ & 0.254 \\
\hline$\geq 2$ & $8(21)$ & $7(12)$ & \\
\hline \multicolumn{4}{|l|}{ Ann Arbor stage } \\
\hline I/II & $3 / 7(26)$ & $10 / 6(27)$ & $>0.99$ \\
\hline III/ IV & $6 / 22(74)$ & $11 / 33(73)$ & \\
\hline \multicolumn{4}{|l|}{ Extranodal involvement } \\
\hline $0 / 1$ & $13(34)$ & $34(57)$ & 0.060 \\
\hline$\geq 2$ & $23(60)$ & $26(43)$ & \\
\hline Missing & $2(5)$ & 0 & \\
\hline \multicolumn{4}{|l|}{ Serum LDH } \\
\hline Normal & $9(24)$ & $23(38)$ & 0.490 \\
\hline Increased & $21(55)$ & $37(62)$ & \\
\hline Missing & $8(21)$ & 0 & \\
\hline \multicolumn{4}{|c|}{ International Prognostic Index } \\
\hline Low/Low-intermediate & $8 / 3(29)$ & $19 / 21(67)$ & 0.007 \\
\hline High-intermediate/High & $18 / 2(53)$ & $16 / 4(33)$ & \\
\hline Missing & $7(18)$ & 0 & \\
\hline \multicolumn{4}{|l|}{ Bone marrow involvement } \\
\hline Absence & $29(76)$ & $39(65)$ & 0.163 \\
\hline Presence & $7(18)$ & $21(35)$ & \\
\hline Missing & $2(50)$ & 0 & \\
\hline \multicolumn{4}{|l|}{ B symptoms } \\
\hline Absence & $20(53)$ & $31(52)$ & $>0.99$ \\
\hline Presence & $18(47)$ & $29(48)$ & \\
\hline \multicolumn{4}{|l|}{ Type } \\
\hline T-cell lymphoma & $17(45)$ & $34(57)$ & 0.302 \\
\hline NK/T-cell lymphoma & $21(55)$ & $26(43)$ & \\
\hline \multicolumn{4}{|l|}{ Subtype } \\
\hline PTCL-NOS & $8(21)$ & $15(25)$ & 0.250 \\
\hline AITL & $3(8)$ & $8(13)$ & \\
\hline ALCL, ALK-negative & $2(5)$ & $9(15)$ & \\
\hline EATL & $3(8)$ & 0 & \\
\hline HSTL & 0 & $1(2)$ & \\
\hline SPTL & $1(3)$ & $1(2)$ & \\
\hline ENKTL & $21(55)$ & $26(43)$ & \\
\hline \multicolumn{4}{|l|}{ Transplantation type } \\
\hline Upfront & $16(42)$ & $29(48)$ & 0.678 \\
\hline Salvage & $22(58)$ & $31(52)$ & \\
\hline \multicolumn{4}{|l|}{ Pre-transplantation response } \\
\hline Complete response & $25(66)$ & $34(57)$ & 0.404 \\
\hline Partial response & $13(34)$ & $26(43)$ & \\
\hline
\end{tabular}


Table 1. Continued

\begin{tabular}{|c|c|c|c|}
\hline Characteristic & TBI (n=38) & Non-TBI (n=60) & p-value \\
\hline \multicolumn{4}{|c|}{ Pre-transplantation PET response } \\
\hline Complete response & $14(42)$ & $17(44)$ & $>0.99$ \\
\hline Partial response & $19(58)$ & $22(56)$ & \\
\hline
\end{tabular}

Values are presented as number (\%). TBI, total body irradiation; ECOG, Eastern Cooperative Oncology Group; LDH, lactate dehydrogenase; PTCL-NOS, peripheral T-cell lymphoma, not-otherwise specified; AITL, angioimmunoblastic T-cell lymphoma; ALCL, anaplastic large cell lymphoma; ALK, anaplastic lymphoma kinase; EATL, enteropathy associated T-cell lymphoma; HSTL, hepatosplenic T-cell lymphoma; SPTL, subcutaneous panniculitis-like T-cell lymphoma; ENKTL, extranodal natural killer/T-cell lymphoma; PET, positron emission tomography.

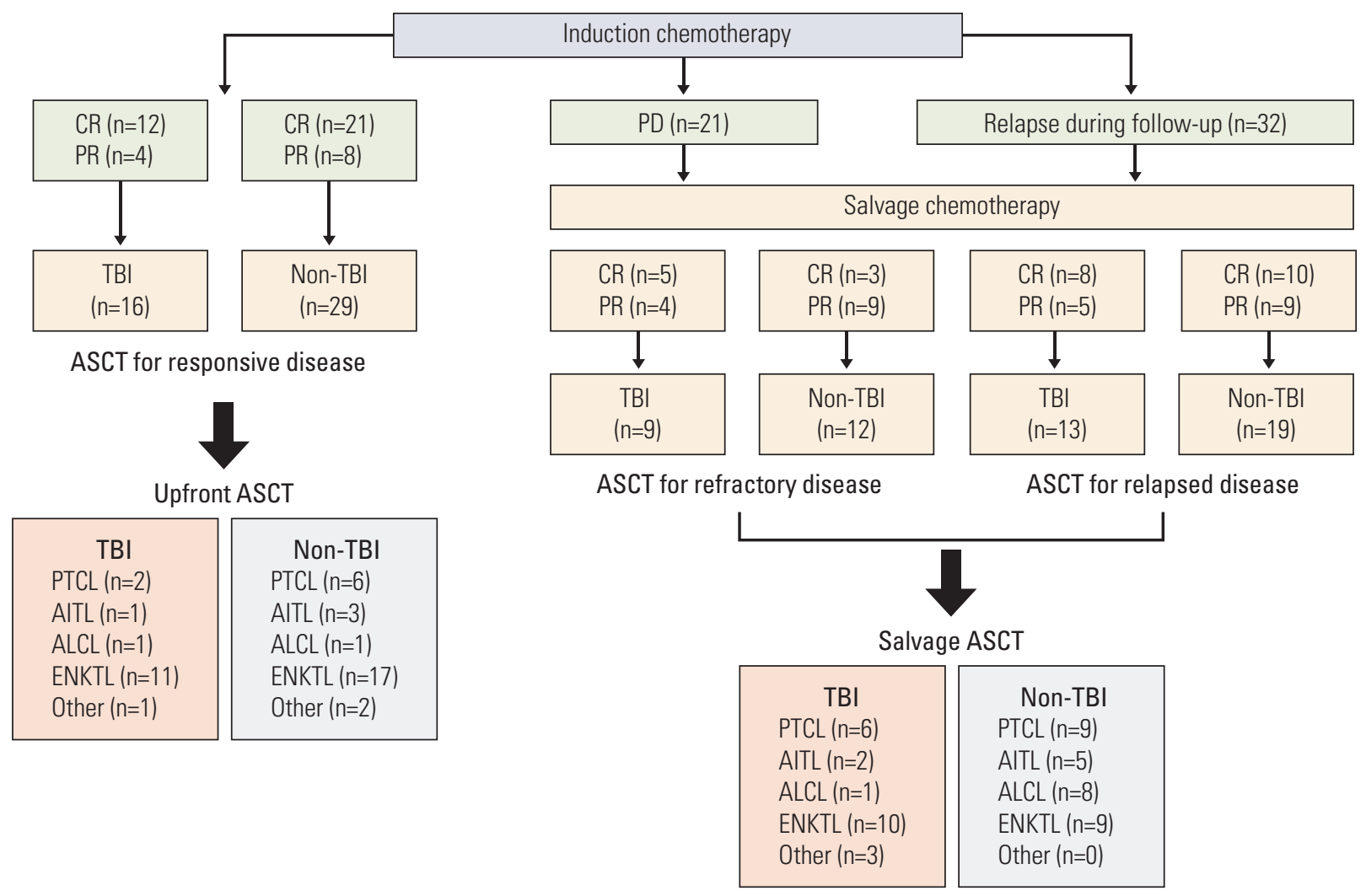

Fig. 1. Classification of patients according to response to induction treatment and time of autologous stem cell transplantation. CR, complete response; PR, partial response; PD, progressive disease; TBI, total body irradiation; ASCT, autologous stem cell transplantation; PTCL, peripheral T-cell lymphoma; AITL, angioimmunoblastic T-cell lymphoma; ALCL, anaplastic large cell lymphoma; ENKTL, extranodal natural killer/T-cell lymphoma.

patients with ENKTL. Patients who showed CR or PR to induction chemotherapy underwent upfront ASCT for treatment of their responsive disease (TBI, $n=16$; non-TBI, $n=29$ ). If the response to induction chemotherapy was progressive disease, the treatment was switched to intensified salvage chemotherapies. These primary refractory patients underwent ASCT in the state of CR or PR (TBI, n=9; non-TBI, $\mathrm{n}=12$ ). Patients who had relapsed disease during follow-up after showing CR or PR to salvage treatments also underwent ASCT (TBI, n=13; non-TBI, $\mathrm{n}=19$ ) (Fig. 1). Salvage 
Table 2. Outcomes and toxicities of conditioning for ASCT

\begin{tabular}{|c|c|c|}
\hline Outcome and toxicity & TBI & Non-TBI \\
\hline \multicolumn{3}{|l|}{ Engraftment and mortality } \\
\hline Neutrophil (day) & $10(8-33)$ & $10(8-29)$ \\
\hline Platelet (day) & $11(9-54)$ & $11(9-37)$ \\
\hline 100-Day mortality & $5(13)$ & $7(12)$ \\
\hline \multicolumn{3}{|l|}{ Infection-related complications } \\
\hline Febrile neutropenia G3 / G4 & $32 / 5$ & $44 / 9$ \\
\hline Proven bacteremia $(-) /(+)$ & $28 / 10$ & $41 / 19$ \\
\hline Fungal infection $(-) /(+)$ & $38 / 0$ & $59 / 1$ \\
\hline Cytomegalovirus antigenemia $(-) /(+)$ & $36 / 2$ & $58 / 2$ \\
\hline \multicolumn{3}{|l|}{ Toxicity according to the Seattle criteria } \\
\hline Stomatitis $\mathrm{G} 1 / 2 / 3 / 4$ & $18 / 16 / 1 / 0$ & $26 / 20 / 2 / 0$ \\
\hline Gastrointestinal toxicity G1/2/3/4 & $17 / 9 / 0 / 0$ & $24 / 13 / 0 / 0$ \\
\hline Pulmonary dysfunction G1/2/3/4 & $1 / 1 / 1 / 0$ & $1 / 2 / 0 / 0$ \\
\hline Central nervous system dysfunction G1/2/3/4 & $0 / 1 / 0 / 0$ & $2 / 0 / 0 / 0$ \\
\hline Heart dysfunction G1/2/3/4 & $1 / 3 / 0 / 0$ & $1 / 2 / 0 / 0$ \\
\hline \multicolumn{3}{|l|}{ Cumulative toxicity score } \\
\hline Low risk $(0-4)$ & $34(89.5)$ & $54(90)$ \\
\hline Intermediate risk (5-8) & $4(10.5)$ & $6(10)$ \\
\hline High risk ( $\geq 9)$ & 0 & 0 \\
\hline
\end{tabular}

Values are presented as median (range) or number (\%). Cumulative toxicity scores are the sum of each grade of toxicity. ASCT, autologous stem cell transplantation; TBI, total body irradiation.

chemotherapies were repeated for these relapsed or refractory patients until achievement of CR or PR (median number of treatment regimen, 2; range, 2 to 4 ).

\section{Toxicities of TBI and non-TBI-based conditioning for ASCT}

The TBI group $(\mathrm{n}=38)$ received a single regimen, etoposide, cyclophosphamide, and TBI conditioning, whereas four conditioning regimens were used in the non-TBI group (BEAM, $\mathrm{n}=15$; Bu-Cy-etoposide, $\mathrm{n}=25$; Bu-Mel-etoposide, $\mathrm{n}=16$; Bu-Mel-Thiotepa, $\mathrm{n}=4$ ). The median time to neutrophil engraftment (> ANC $500 / \mu \mathrm{L}$ ) and platelet engraftment (> 20,000/ $\mu \mathrm{L}$ ) was 10 and 11 days after stem cell infusion, respectively, in both groups (Table 2). The median hospitalization period was 16 days (range, 8 to 287 days) in the TBI group and 15 days (range, 9 to 134 days) in the non-TBI group. Most cases of hospitalization longer than 60 days were related to early relapse rather than transplantationrelated morbidity. In the TBI group five patients died within 100 days from ASCT $(13 \%, 5 / 38)$, and all deaths were associated with disease progression immediately after ASCT. In the non-TBI group, seven patients $(12 \%, 12 / 60)$ died within 100 days, and two deaths were due to septic shock and the remaining five deaths were related to disease progression combined with infection. Most patients suffered from febrile neutropenia, but hematologic and non-hematologic toxicities were manageable, and the median cumulative toxicity score according to Seattle criteria was estimated as 2 (range, 0 to 7 ) in both groups. Thus, there were no cases of high-risk cumulative toxicity (Table 2).

\section{Comparison of PFS between TBI and non-TBI group}

As the deaths and disease relapse occurred early after ASCT, the curves of PFS and OS after ASCT showed a steep drop within 1 year after ASCT. The comparison of OS between the TBI and non-TBI groups showed no significant difference, although the survival curve for the TBI group showed a plateau (Fig. 2A). The TBI group also showed a trend of better PFS than the non-TBI group; however, the difference was not significant (Fig. 2B). This pattern of survival difference was also observed in patients who underwent upfront ASCT as well as in the salvage setting (Fig. 2C and D). In the subgroup analysis in patients with ENKTL and other remaining other T-cell lymphoma patients, a trend of better PFS was observed in the TBI group compared with the non-TBI group, although without statistical significance (Fig. 2E and F). 
A
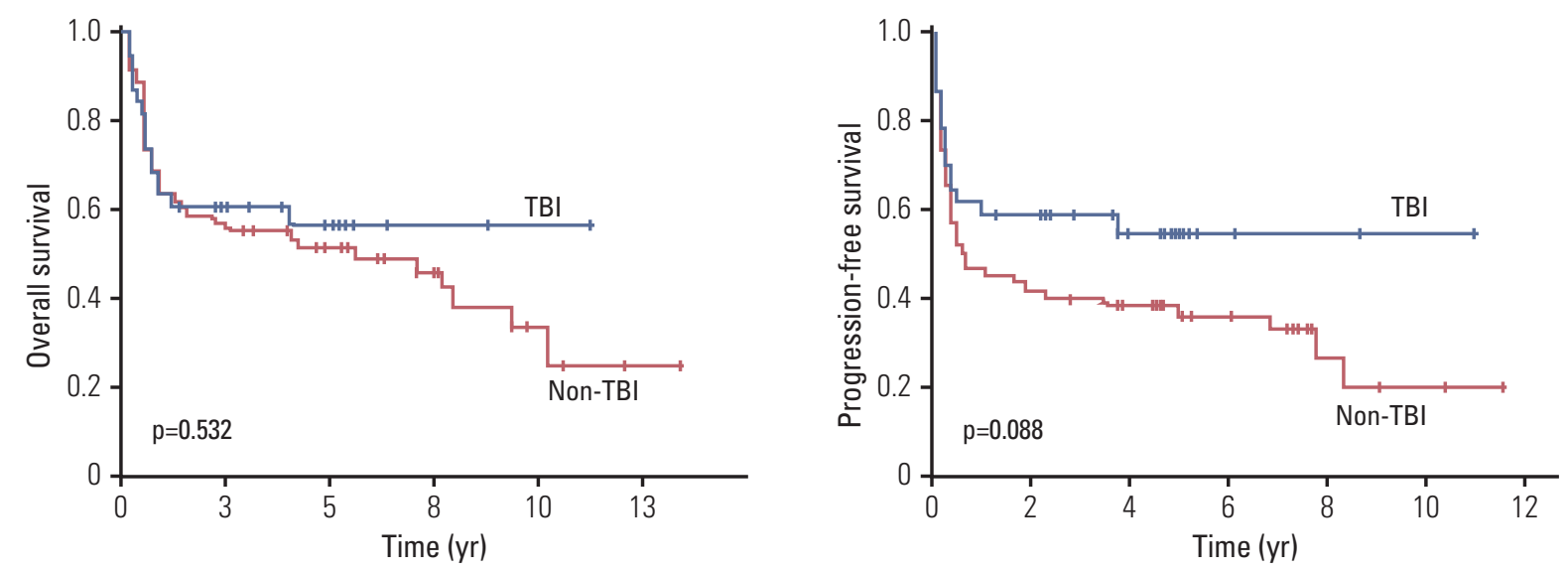

C
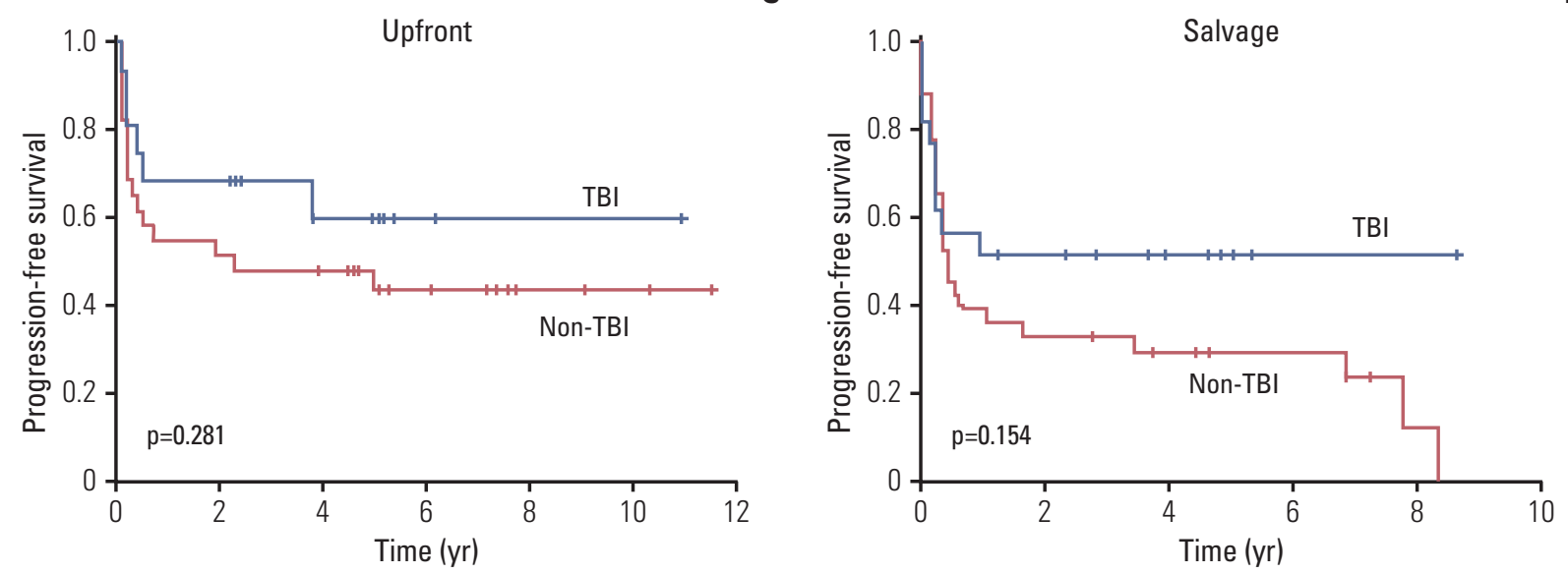

E
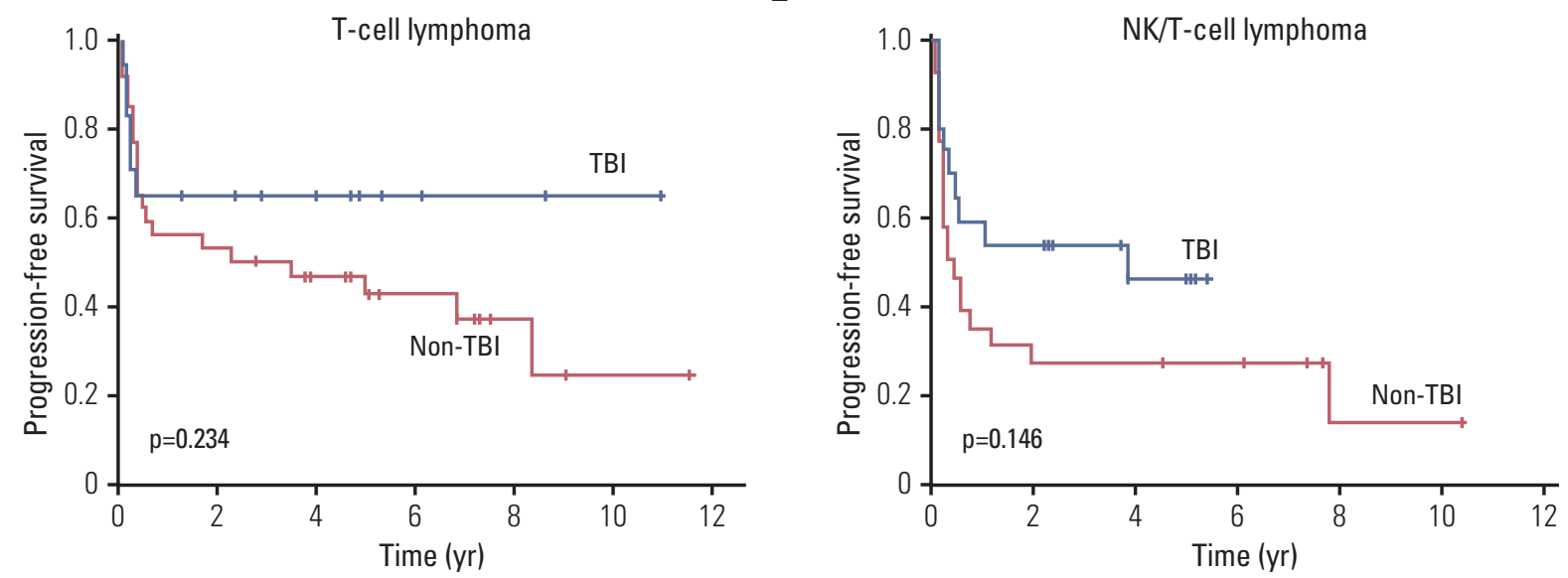

Fig. 2. (A, B) Comparison of overall and progression-free survival according to the use of total body irradiation (TBI). (C, D) Comparison of progression-free survival between TBI and non-TBI group in upfront and salvage autologous stem cell transplantation. (E, F) Comparison of progression-free survival between TBI and non-TBI group in T-cell lymphomas and extranodal natural killer (NK)/T-cell lymphoma. 
A
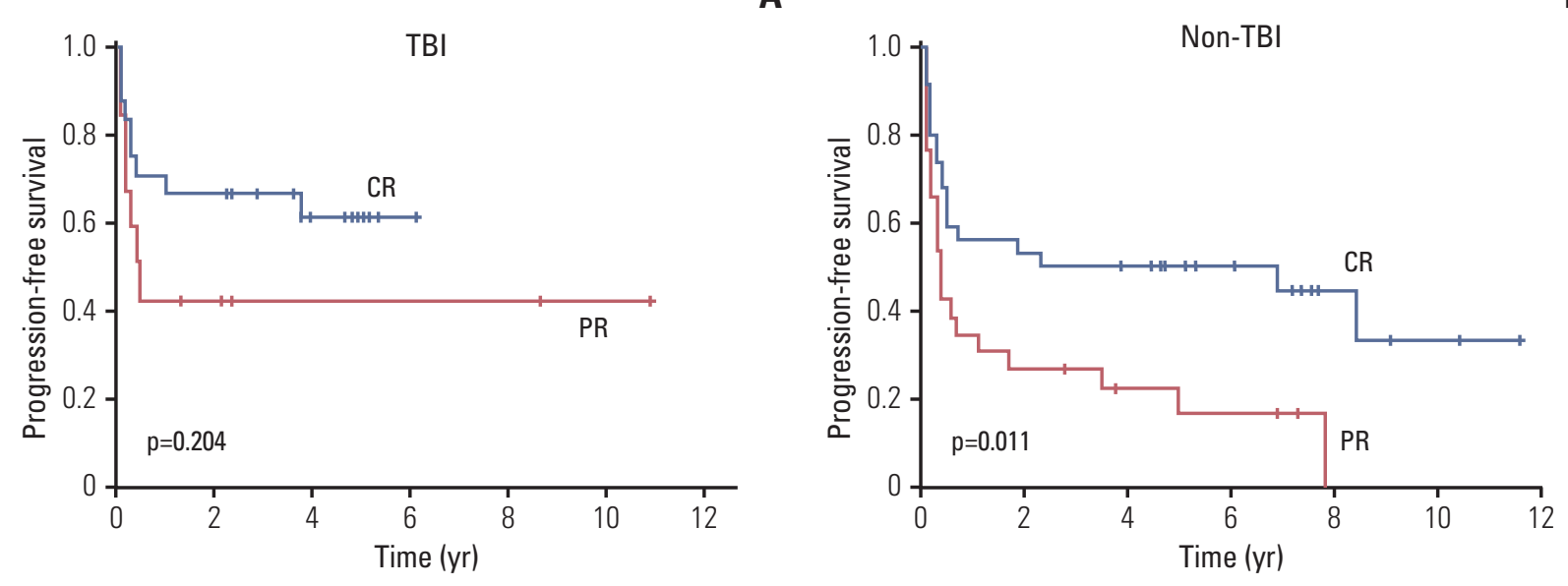

C
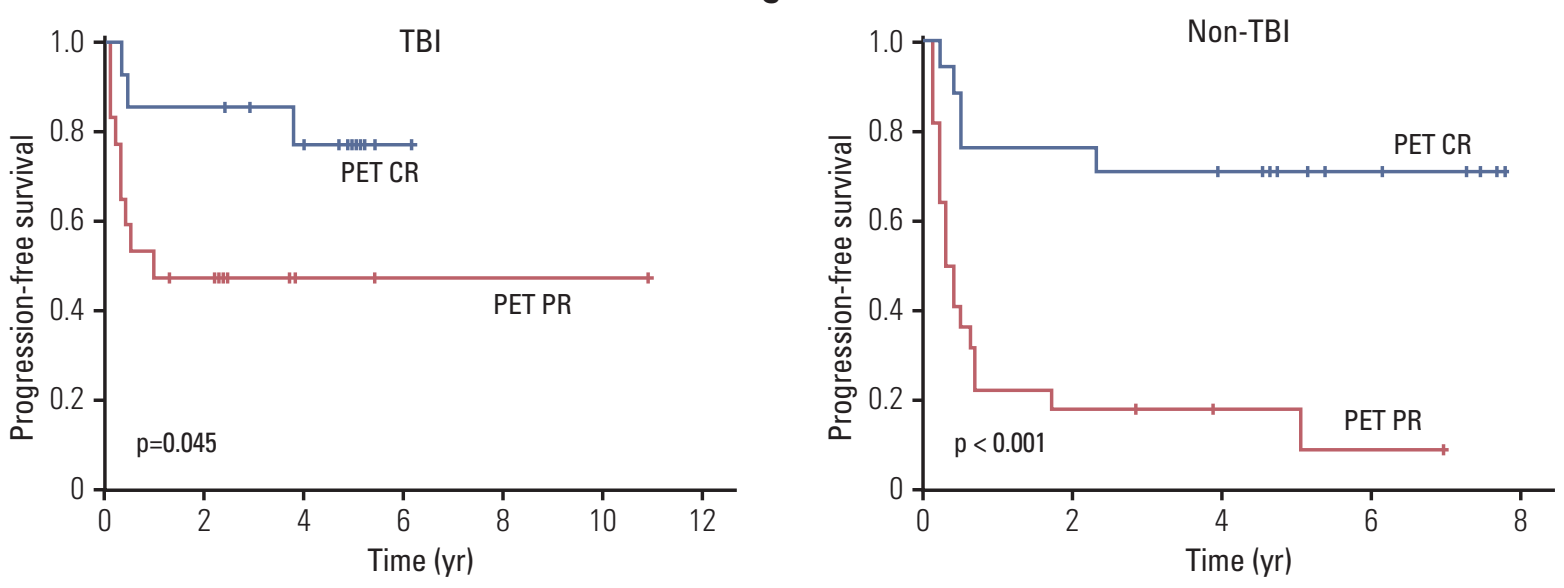

E
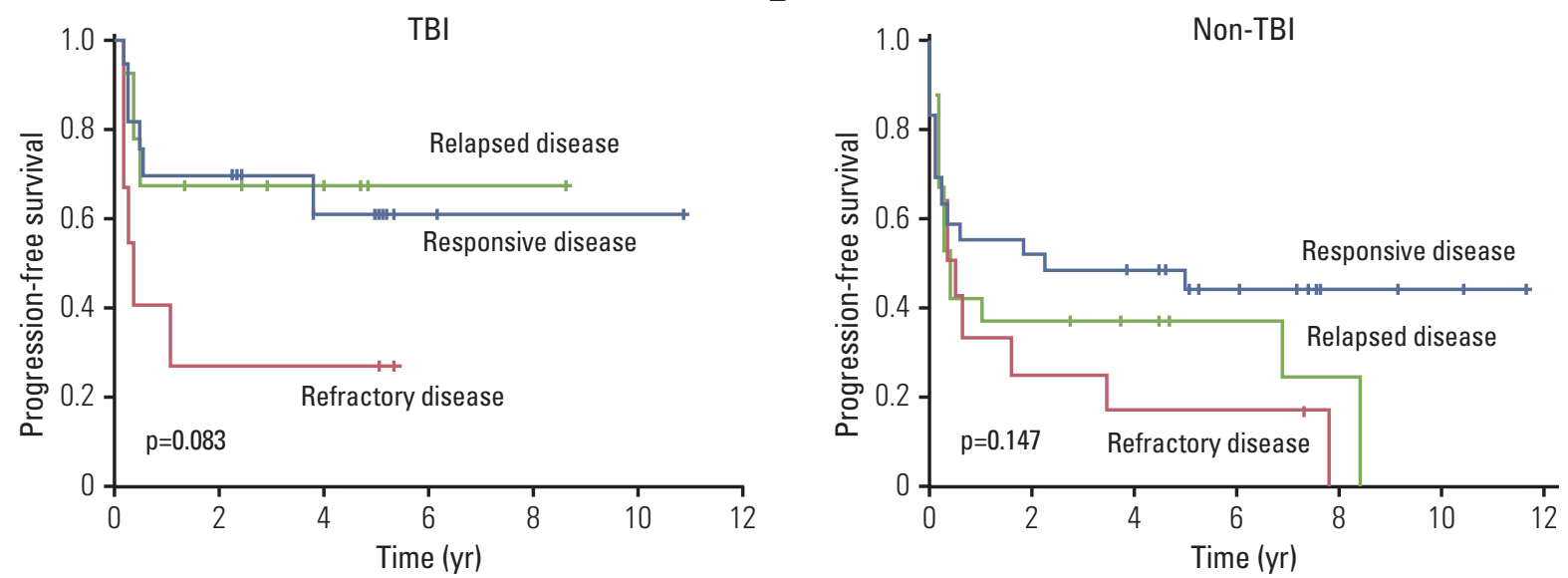

Fig. 3. (A, B) Comparison of progression-free survival between patients with pre-transplantation complete response (CR) and partial response (PR) in the total body irradiation (TBI) and non-TBI group. (C, D) Association of pre-transplantation $\mathrm{CR}$ assessed by positron emission tomography (PET) computed tomography and progression-free survival in the TBI and non-TBI group. (E, F) Comparison of progression-free survival according to the response to induction treatment in the TBI and non-TBI group. 
A
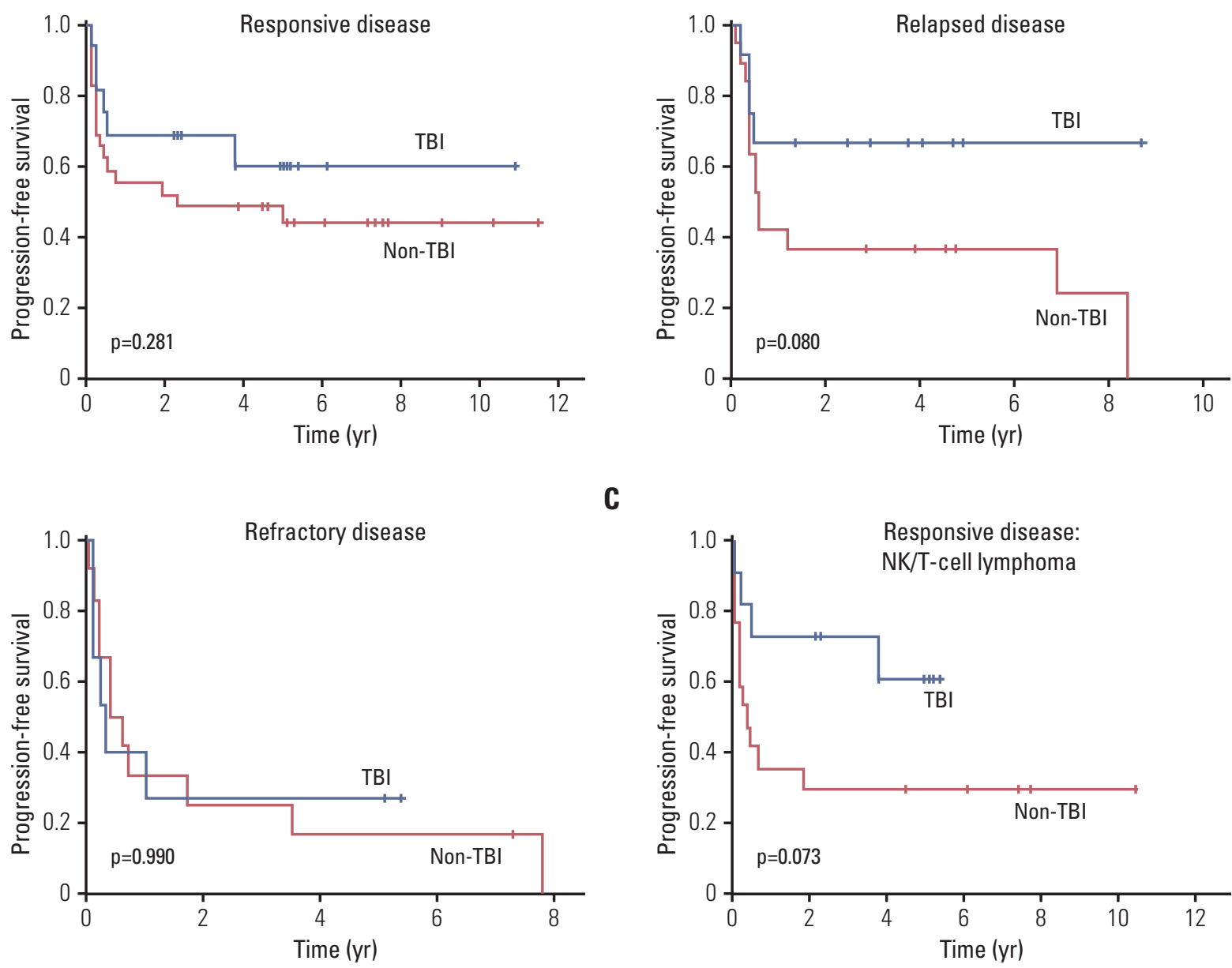

C

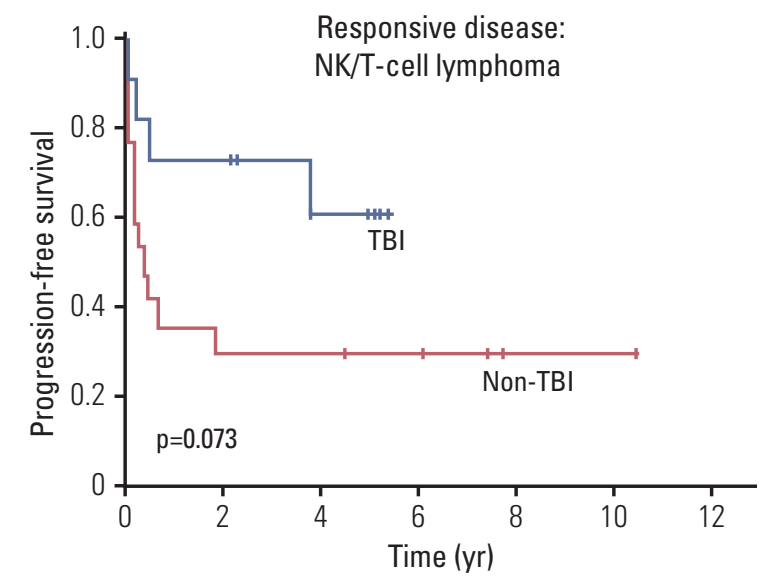

$\mathbf{E}$
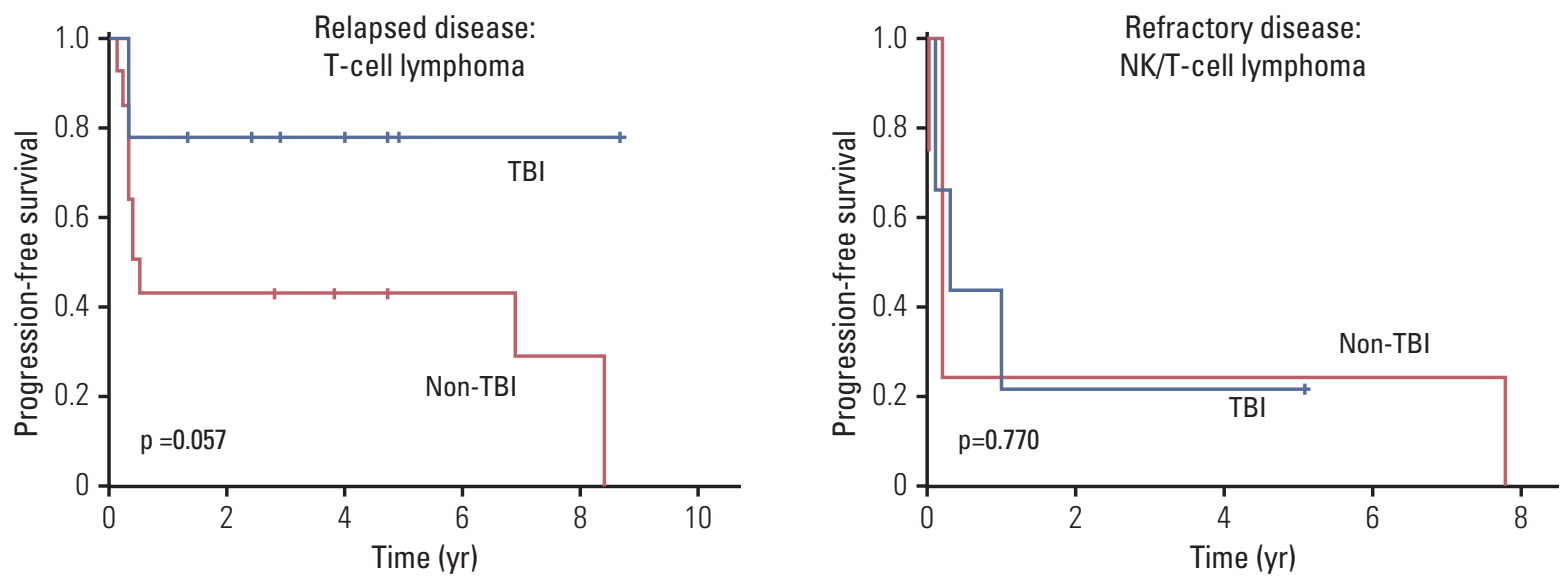

Fig. 4. (A-C) Comparison of progression-free survival of patients with responsive, refractory, and relapsed disease between the total body irradiation (TBI) and non-TBI groups. (D, E) The TBI group showed a trend of better progression-free survival than the non-TBI group in extranodal natural killer (NK)/T-cell lymphoma with responsive disease and T-cell lymphoma with relapsed disease. (F) No difference in progression-free survival of refractory disease was observed in patients with extranodal NK/T-cell lymphoma. 


\section{Pre-transplantation disease status and outcomes of ASCT}

The pre-transplantation disease status was evaluated by $\mathrm{CT}$, and a review of medical records showed that all patients achieved CR or PR before ASCT (Table 1). Patients with pretransplantation CR showed better PFS after ASCT than patients with PR, and a significant difference in PFS was observed in the non-TBI group (Fig. 3A and B). Patients with available PET-CT scan data were dichotomized according to PET CR and PET PR, and the pre-transplantation disease status based on PET-CT showed significant association with PFS after ASCT (Fig. 4B and E). The clinical situation of patients could also be classified according to three groups based on their response to induction treatment: ASCT for responsive disease (upfront ASCT performed for patients with CR or PR after induction treatment); ASCT for refractory disease (ASCT performed for patients who were refractory to induction treatment but achieved CR or PR after salvage treatment); and ASCT for relapsed disease (ASCT performed for patients with relapsed disease). The median time between diagnoses to the date of ASCT was 0.4 and 0.6 years in ASCT for responsive disease and for refractory disease, respectively. On the other hand, all patients who underwent ASCT after relapse had disease sensitive to chemotherapy, and the median time to ASCT was 1.5 years. ASCT for responsive disease was designated as upfront ASCT whereas ASCT for refractory or relapsed disease was designated as salvage ASCT (Fig. 1). Patients who underwent ASCT for responsive disease after showing CR or PR to induction treatment showed better PFS than patients who were refractory to induction treatment, although the difference was not statistically significant (Fig. 3E and F). In the subgroup analyses with responsive and relapsed disease, a trend of better PFS was observed in the TBI group compared to the non-TBI group, although the difference was not statistically significant (Fig. 4A and B). However, there was no difference in PFS regardless of TBI use in case of refractory disease (Fig. 4C). This difference in outcome according to the use of TBI was also shown in responsive disease with ENKTL as well as relapsed disease with other T-cell lymphomas (Fig. 4D and E). However, the PFS of refractory disease was not different in patients with ENKTL (Fig. 4F).

\section{Discussion}

In this study, the TBI group showed a trend of better PFS than the non-TBI group, although without statistical significance $(p=0.088)$ (Fig. 2B). These trends were also shown in upfront and salvage ASCT because patients who underwent
TBI-containing conditioning showed a plateau on the PFS curve (Fig. 2C and D). The comparison of TBI and non-TBI groups in ENKTL and other T-cell lymphomas also showed a trend of better PFS in the TBI group compared with the non-TBI group (Fig. 2E and F). Hematologic and non-hematologic toxicities were manageable, and were not significantly different between the two groups (Table 2). There were no cases of engraftment failure and the median cumulative toxicity score according to the Seattle criteria was 2. The 100-day mortality rate was similar in both groups, and the main cause of death was related to disease progression rather than transplantation-related morbidity. Thus, our study showed that a TBI-containing conditioning regimen might be feasible for mature T- and NK-cell lymphoma patients in terms of efficacy and toxicity.

The pre-transplantation disease status also showed significant association with the survival outcome of ASCT. In our study achievement of CR before ASCT showed association with better survival outcomes. In particular, patients in CR state determined by PET-CT scan showed significantly better outcome than patients with PR (Fig. 3A and B). Our findings were supported by a recent retrospective study of 134 patients who underwent ASCT in the upfront or salvage setting; patients with CR at the time of ASCT showed significantly better OS and PFS than patients with PR [18]. Therefore, the efficacy of ASCT might be lower than expected when residual disease is detected on PET-CT. In such cases, other treatment modalities including allogenic stem cell transplantation might be a viable option, although a previous study reported that the application of allogeneic stem cell transplantation did not provide a survival advantage over ASCT in patients with PR [18]. However, in our study the survival difference between pre-transplantation CR and PR was less prominent in the TBI group compared to the nonTBI group (Fig. 3C and D). Because the negative impact of PR before ASCT might be reduced by the application of TBIbased conditioning, this finding might support the efficacy of TBI-based conditioning. However, evaluation with further prospective studies will be required using a larger study population.

In the comparison of survival outcomes according to clinical situation, patients with refractory disease showed worse PFS than patients with responsive or relapsed disease despite undergoing ASCT in the state of CR or PR after salvage treatment (Fig. 3E and F). This implied that ASCT could not provide prolonged survival in patients with primary refractory to induction treatment even though they achieved response after salvage therapy. Therefore, the role of ASCT in patients with suboptimal response to induction chemotherapy might be limited and other treatment strategies such as clinical trials or allogeneic stem cell transplantation should be considered for patients with primary refractory disease. In addition, 
the survival outcome of patients undergoing upfront ASCT for responsive disease was not significantly different from that of patients who underwent ASCT after disease relapse, particularly in patients in the TBI group (Fig. 3E). This finding is not consistent with results of a recent study from the Swedish Lymphoma Registry reporting an association of upfront ASCT with a better OS and PFS compared to patients treated without ASCT [19]. However, these results should be interpreted with caution due to the possibility of selection bias for upfront ASCT. Patients at high risk of relapse might be more likely to undergo upfront ASCT than patients at low risk. Nevertheless, the subgroup analyses with responsive and relapsed disease showed a trend of better PFS in the TBI group compared with the non-TBI group (Fig. 4A and B). This difference in outcome according to the use of TBI was also shown in ENKTL and other T-cell lymphomas (Fig. 4D and E). Thus, TBI containing conditioning might be useful for upfront and salvage ASCT in patients with mature T- and NK-cell lymphoma if those patients show CR or PR to induction treatment.

\section{Conclusion}

In conclusion, the survival outcomes and toxicities of patients receiving TBI-containing conditioning regimen were comparable to those of other conditioning without TBI. In addition, patients in the state of responsive disease to induction treatment might gain a survival advantage with ASCT after TBI containing conditioning. However, a survival benefit was not observed in patients who were refractory to induction treatment. Likewise, assessment of the CR state by PET-CT immediately before ASCT could lead to prediction of better survival outcomes of patients undergoing ASCT regardless of TBI use.

\section{Conflicts of Interest}

Conflict of interest relevant to this article was not reported.

\section{Acknowledgments}

This study was supported by grants from the Samsung Biomedical Research Institute (GL1B30311), and the National Research Foundation of Korea's (NRF) Basic Science Research Program (NRF-2014R1A2A1A11049853), which is funded by the Ministry of Education, Science and Technology.

\section{References}

1. Philip T, Guglielmi C, Hagenbeek A, Somers R, Van der Lelie $\mathrm{H}$, Bron D, et al. Autologous bone marrow transplantation as compared with salvage chemotherapy in relapses of chemotherapy-sensitive non-Hodgkin's lymphoma. N Engl J Med. 1995;333:1540-5.

2. Rudiger T, Weisenburger DD, Anderson JR, Armitage JO, Diebold J, MacLennan KA, et al. Peripheral T-cell lymphoma (excluding anaplastic large-cell lymphoma): results from the Non-Hodgkin's Lymphoma Classification Project. Ann Oncol. 2002;13:140-9.

3. A clinical evaluation of the International Lymphoma Study Group classification of non-Hodgkin's lymphoma. The NonHodgkin's Lymphoma Classification Project. Blood. 1997;89: 3909-18.

4. d'Amore F, Relander T, Lauritzsen GF, Jantunen E, Hagberg $\mathrm{H}$, Anderson $\mathrm{H}$, et al. Up-front autologous stem-cell transplantation in peripheral T-cell lymphoma: NLG-T-01. J Clin Oncol. 2012;30:3093-9.

5. Foss FM. Treatment strategies for peripheral T-cell lym- phomas. Best Pract Res Clin Haematol. 2013;26:43-56.

6. Jantunen E, Wiklund T, Juvonen E, Putkonen M, Lehtinen T, Kuittinen $\mathrm{O}$, et al. Autologous stem cell transplantation in adult patients with peripheral T-cell lymphoma: a nation-wide survey. Bone Marrow Transplant. 2004;33:405-10.

7. Yang P. TEST: a public health and law enforcement collaboration to increase HIV / AIDS prevention in Westchester County, New York. J Public Health Manag Pract. 2009;15:118-22.

8. Feyler S, Prince HM, Pearce R, Towlson K, Nivison-Smith I, Schey $\mathrm{S}$, et al. The role of high-dose therapy and stem cell rescue in the management of T-cell malignant lymphomas: a BSBMT and ABMTRR study. Bone Marrow Transplant. 2007; 40:443-50.

9. Reimer P, Rudiger T, Geissinger E, Weissinger F, Nerl C, Schmitz N, et al. Autologous stem-cell transplantation as firstline therapy in peripheral T-cell lymphomas: results of a prospective multicenter study. J Clin Oncol. 2009;27:106-13.

10. Yhim HY, Kim JS, Mun YC, Moon JH, Chae YS, Park Y, et al. Clinical outcomes and prognostic factors of up-front autolo- 
gous stem cell transplantation in patients with extranodal natural killer/T cell lymphoma. Biol Blood Marrow Transplant. 2015;21:1597-604.

11. Yin J, Wei J, Xu JH, Xiao Y, Zhang YC. Autologous stem cell transplantation as the first-line treatment for peripheral $\mathrm{T}$ cell lymphoma: results of a comprehensive meta-analysis. Acta Haematol. 2014;131:114-25.

12. Gkotzamanidou M, Papadimitriou CA. Peripheral T-cell lymphoma: the role of hematopoietic stem cell transplantation. Crit Rev Oncol Hematol. 2014;89:248-61.

13. Shustov A. Controversies in autologous and allogeneic hematopoietic cell transplantation in peripheral T/NK-cell lymphomas. Best Pract Res Clin Haematol. 2013;26:89-99.

14. Swerdlow SH, Campo E, Harris NL, Jaffe ES, Pileri SA, Stein $\mathrm{H}$, et al. WHO classification of tumours of haematopoietic and lymphoid tissues. 4th ed. Lyon: IARC Press; 2008.

15. Cheson BD, Horning SJ, Coiffier B, Shipp MA, Fisher RI, Connors JM, et al. Report of an international workshop to standardize response criteria for non-Hodgkin's lymphomas. NCI
Sponsored International Working Group. J Clin Oncol. 1999;17:1244.

16. Moon SH, Cho SK, Kim WS, Kim SJ, Ahn YC, Choe YS, et al. The role of 18F-FDG PET / CT for initial staging of nasal type natural killer/T-cell lymphoma: a comparison with conventional staging methods. J Nucl Med. 2013;54:1039-44.

17. Bearman SI, Appelbaum FR, Buckner CD, Petersen FB, Fisher LD, Clift RA, et al. Regimen-related toxicity in patients undergoing bone marrow transplantation. J Clin Oncol. 1988;6:15628.

18. Beitinjaneh A, Saliba RM, Medeiros LJ, Turturro F, Rondon G, Korbling $\mathrm{M}$, et al. Comparison of survival in patients with $\mathrm{T}$ cell lymphoma after autologous and allogeneic stem cell transplantation as a frontline strategy or in relapsed disease. Biol Blood Marrow Transplant. 2015;21:855-9.

19. Ellin F, Landstrom J, Jerkeman M, Relander T. Real-world data on prognostic factors and treatment in peripheral T-cell lymphomas: a study from the Swedish Lymphoma Registry. Blood. 2014;124:1570-7. 